\title{
Scalable Conversational Intelligence for Post-COVID Health Consultation Using Flask Library and Support Vector Machine
}

\author{
Uday Kumar Adusumilli'i , Druthi R², Malvika K², Megha N B2, Prof. R Geetha ${ }^{3}$
}

${ }^{1}$ Product Support Analyst, Associate, Infor, Bangalore, Karnataka, India

${ }^{2}$ Students, Department of Information Science and Engineering, East Point College of Engineering and Technology, Bangalore, Karnataka, India

${ }^{3}$ Professor, Department of Information Science and Engineering, East Point College of Engineering and Technology, Bangalore, Karnataka, India

\section{ABSTRACT}

In this paper, we put forth a model for natural language processing (NLP)-based Multilingual Conversational Bot in order to provide economic primary healthcare education, information, and advice to chronic patients. Telemedicine and Intelligent Communication Systems can be employed by Medical Practitioners, at scale, as part of their communication strategy during the recent Coronavirus outbreak in order to discourage contagion in the post-covid era. Such systems have the potential to propagate vital, valid, authenticated, up to date, information, and support, straight to the masses. The prime intention of the proposed system is to enable our already strained medical infrastructure to scale up its serviceability exponentially. Deep Learning based Conversational Intelligence Systems offer the potential for rapid and radically transforming patient care from an in-person to a remote experience. We introduce a novel computer application that enables healthcare professionals to delegate certain functions. The system was developed precisely, and it has been exhaustively trained to interact with patients alike human beings. The proposed system is based on a serverless architecture, aggregating information from a healthcare professional and providing ideas on preventative measures, home remedies, interactive counseling sessions, healthcare tips, and symptom information on diseases prevalent in rural areas of India. We leverage the resources of the Google Cloud Platform (GCP) for the provision of the said services in India, which will increase the availability of healthcare information to patients, and to leverage the immense potential of AI to eliminate the gap between the demand for healthcare services and the supply of healthcare providers.

Keywords : Telemedicine, Intelligent Communication Systems, Coronavirus, Contagion, Medical Infrastructure, Deep Learning Based Conversational Intelligence Systems, Multilingual Conversational Bot, Natural Language Processing (NLP), Economic Primary Healthcare, Serverless Architecture, Google Cloud Platform (GCP), Artificial Intelligence

Copyright: (C) the author(s), publisher and licensee Technoscience Academy. This is an open-access article distributed under the terms of the Creative Commons Attribution Non-Commercial License, which permits unrestricted non-commercial use, distribution, and reproduction in any medium, provided the original work is properly cited 


\section{INTRODUCTION}

As a training tool, the system uses information from different online sources and databases to gather medical information. The application process is designed to take questions from patients and to provide answers based on the questions that they ask and symptoms that they have. As a result, we approached this particular topic with the aim of making it more accessible for patients who are undergoing medical treatment, as well as those who are seeking information about the causes of their symptoms. In addition, we chose this topic to become more responsive to queries that users pose as input queries. For this reason, the system was built to take advantage of techniques from natural language processing to provide a response to the data. Natural language processing is the study of the processing of human language. As a result, this allows us to comprehend and prepare queries for users based on a set of defined rules. These were further explained to explain how the task will be performed and how the generated output will be displayed. With this system, users will be able to interact with the computer using natural language as part of the interaction process. Taking advantage of the extensive amount of information available on the internet, the chatbot can provide the user with accurate and valuable information based on what the user needs. There are several ways the chatbot can be used, for instance as virtual assistants, for customer service, or for online training. Consequently, the user will not only have the capability of asking questions but also get the opportunity to chat with medical professionals in regards to their health issues. The user can search for words containing critical information, which is then used to determine the kind of medical problems that are being experienced through the input of their words. Additionally, our system also feeds in, and processes information from medical and healthcare QA forums, thus deepening it's understanding of the subject and providing more accurate and robust information to the users. Observing and answering similar questions asked by medical professionals may allow the app to learn from and answer similar questions.

In the current version of the chatbot, it is only possible to conduct conversations using text as a medium that can be easily readable. There is however a possibility to make this system more robust in the future to also handle oral conversations.

Among 190 countries listed in the World Health Report produced by the World Health Organization, India's healthcare system is ranked 112 out of 190 . India faces one of its biggest challenges as a country from the perspective of meeting the healthcare needs of its rapidly growing population with good quality and affordable healthcare services. Due to the inaccessibility of health facilities, especially in rural areas of India, and the difficulties of obtaining transport methods, patients either postpone treatment or choose medical facilities that are closer but may not be as effective or efficient as those that are closer but do not match their medical needs. Telemedicine, which is a platform of connecting patients with healthcare providers and healthcare information in order to provide timely medical care, access, and quality treatment to patients, has come into play in order to achieve more efficient means of providing timely medical care. Since the recent COVID-19 pandemic has created an air of social distancing, especially in India among those with chronic illnesses, this will definitely remain a disadvantage for the population when it comes to accessing healthcare facilities for a long time to come.

The National Health Mission, in releasing the data in light of the ongoing COVID-19 outbreak in India, has shown that there has been a fall in the number of patients reporting other acute illnesses. There is evidence to suggest that hospitalizations are reduced as a result, rather than due to a lack of illnesses, as a result of poor healthcare access. A conversational artificial intelligence platform can offer healthcare providers diagnostic and treatment services without 
requiring personal visits, promoting social distance, and minimizing the likelihood of the transmission of COVID-19.

With the blossoming of digitization in the current decade, chatbots powered by Artificial Intelligence (AI) are playing a leading role in commoditizing the function of a virtual assistant that can manage a conversation so it can be conducted either verbally or through text transmission. In order to provide the user with knowledge, perform actions, and make recommendations accordingly to their needs, it uses voice queries. The language you use, the frequency of searches, and your preferences are adaptable over time according to your individual usage. In our new digital economy, conversational bots that communicate via voice, text, and/or chat have the potential to play a principal role in overcoming the current barriers that are preventing primary healthcare from being affordable, accessible, and resilient. Virtual assistants have been observed to have been penetrating the nooks and crannies of the world since the advent of artificial intelligence. In the near future, the emergence of intelligent communication systems based on conversational AI will provide a significant opportunity to utilize instant, personalized services, and advanced, effective communication. It is possible to talk to a voice assistant via a natural language interface which uses natural language to communicate. Healthcare must be customized in order to make use of voice technology in order to be beneficial to patients. Healthcare voice assistants are primarily used by patients and physicians, and two of the two constituencies are likely to use them. As a result, physicians have access to and can record the patient's data using the applications. It is a cheaper alternative, as far as the patient is concerned; AI-enabled virtual assistants that can offer $24 / 7$ assistance to a wide variety of patients. Such powerful virtual tools make virtual communication and conversing much more accessible and accessible to people with chronic diseases, patients with disabilities, as well as patients living in rural and backward areas. Using these systems has many advantages: reducing the amount of time physicians need to spend on patient care, improving data security, allowing access to healthcare information on demand and making healthcare more accessible and affordable for all through their intuitive interfaces.

In addition to the above, the paper discusses how Conversational Intelligence can be integrated into the Telemedicine System. An important part of the solution consists of implementing a multilingual Voice Application designed for Natural Language Processing that can be used by chronic patients and women in need of antenatal care to receive education and advice regarding primary healthcare. In order to process the user's speech, it converts the speech into text that is then sent to a natural language processor for processing, and then the output is turned into speech again, which is then returned to the user. In addition to providing healthcare for the most prevalent diseases in rural India, we have also focused on the health of women. By providing medical advice, home remedies, healthcare tips, symptoms, and diet recommendations based on the location and circumstances of the user, we enable our application to perform the role of a doctor. There is nothing better than prevention, which is why our software and technology will add immense value and be extremely beneficial to those who need a personal healthcare assistant or a personal health assistant.

\section{RELATED WORK}

There have been a number of text-based humancomputer interfaces designed, like ELIZA, which imitates the thoughts of a psychotherapist, then PARRY, which imitates the thoughts of a paranoid patient. Researchers Raij et al. experimented with virtual interactions between human beings and real human beings in a medical consultation scenario where each was compared to the real human. There is a similarity between the results of the virtual and real 
interaction contexts. A. This article summarizes the work undertaken by Fadhils and colleagues in their research on the use of intelligent conversational systems to interact with adult populations to gather health information, monitoring their health condition on a continuous basis, especially after they have left a hospital. A medical recommendation system has been developed by Amato and co-workers in which an interactive medical physician performs the duty of interacting with the user. Pharmabot is a new chatbot interface, designed to function as an online paediatric health consultation service, providing clients with useful information on generic medications for children.

Divya Madhu et al suggested a model for this problem in their study. It uses Artificial Intelligence technology in order to help clinicians establish what type of treatment should be applied to the disease of a patient by utilizing AI. Several treatments are available on the market, so it's likely that nobody will be able to get hold of the treatment that is most suitable for their particular disease. We intend to tackle this problem using AI, which has been proven to be able to accurately predict disease symptoms from a set of data. Identifying the correct health condition and showing the treatments that are applicable is the system's job. The study was conducted by Divya and her collaborators. Using artificial intelligence tools, we have proposed a selfdiagnosis chatbot to diagnose certain illnesses. Ignorance of the conditions, treatment or diagnosis of illnesses appeared in the database. In order for the AI system to predict the disease correctly and with reliability, it will require several diseases and different therapies. An algorithm checks the age and symptoms along with a multitude of other factors and analyzes the data in order to provide the correct advice and diagnosis.

This study was carried out by Amiya et al. Doc-Bot was created to predict the disease and prescribe medicine based on the predicted illness. A small application was developed here to say the patient's symptoms. An account is created by the user with a username and password and, at the most, a unique identifier is assigned to the account. In addition, it is expected that blanks in the statements will be filled in when queries are asked and the principal criterion to fix the disease of the algorithm is the age of the individual. It is possible for other reviewers to be involved once they locate the disease which could then be clarified by other's reviews as well as asking multiple questions to clarify the efficacy of the drug and its availability.

Using a Google API, Rashmi and Neeta built a medical chatbot that works in a similar fashion to a virtual doctor. It uses voice-text to convert it into text-voice and renders it as a social chatbot. As a result, NLP is used in this area to fulfill the purpose of changing the interface with this type of technology. It is, therefore, necessary to establish a virtual platform for patient-doctor communication in order to be able to provide medical information and diagnose problems. In order to develop the proposed system, we have used their work as a starting point.

Ranoliya et. et al. proposed a model in which a response is created using an API interface, which is generally used to create a chatbot that converts speech into text and facilitates the creation of a virtual hospital platform. When diagnosing diseases using the FAQs, several questions are asked, and then the appropriate course of treatment is decided. As a result, medicine was given. This was then reviewed by feedback and questions to ensure that the medicine was supplied precisely.

Agnel, Jenila, and Sagar developed a machine learning algorithm to predict tourist spots and the information associated with them by analyzing social media data. These systems help the tourism industry figure out what kind of experiences visitors can have and how they can best meet the desires of those visitors. Several algorithms have been used to perform this task, including KNNs, Kernel Density Estimation, Random Forests, and XG Boosts. XG Boost is the bestperforming algorithm out of the three, as measured 
by the results that came out of the study. As Lucas et al. suggest, the proposed approach actually works quite nicely. In this study, leaf spectral data were used to estimate the amount of macro- and micronutrients measured in the laboratory. KNN and ANN algorithms were used, as well as RF algorithms in order to obtain the accuracy, which was observed to perform the best.

Shikha and Imran developed a chatterbot based on Artificial Intelligence Markup Language (AIML), which takes the voice of the user to train the model and recognize the words and phrases spoken by the user. The task of understanding and recognizing spoken language is carried out with the help of natural language processing. The study was carried out by Pryss et al. An architecture was proposed as a point of reference for various psychological and medical scenarios that would be able to serve as a basis for further research. There is a need for more collaboration between medical and technical experts in order to improve the quality of conversational intelligence solutions. The results of this study highlight the key elements that may serve as the foundation for this collaboration. It was proposed by Xuewen, Xiaoping, John that using annual Loebner competition data as training data for an SVM model for human and bot classification in a text-based communication system could be helpful. We use appropriate delays between messages and entropy normalized values to support the visibility of each piece of the conversation. As a result of the simulations, it was shown that SVM is an effective way of classifying the data coming from chatbots.

\section{SYSTEM ARCHITECTURE}

Essentially, there are three main components to our system: a client, a server, and the content acquisition portion. During the development of the architecture, an object-access protocol based simple object access protocol (SOAP) was used for the server application component (Web service) of the architecture. (Black
Box) Approach: This approach completely isolates the client from interacting with the internal workings of the web service and their interactions with the web service, whereas White Box Approach: This approach allows the client to interact with the distribution and the inner workings are not necessary to the client. Fig. 1 shows how the process works. A SOAP message pack is composed of a set of messaging messages which are formatted in a XML format using extensible markup language (XML). Due to their text based format, the packs can be used by a wide range of clients and platforms. It contains a processor for the voice recognition module that is located on the client side. Here is the module you need to be able to send and receive plain text messages between the client and the server.

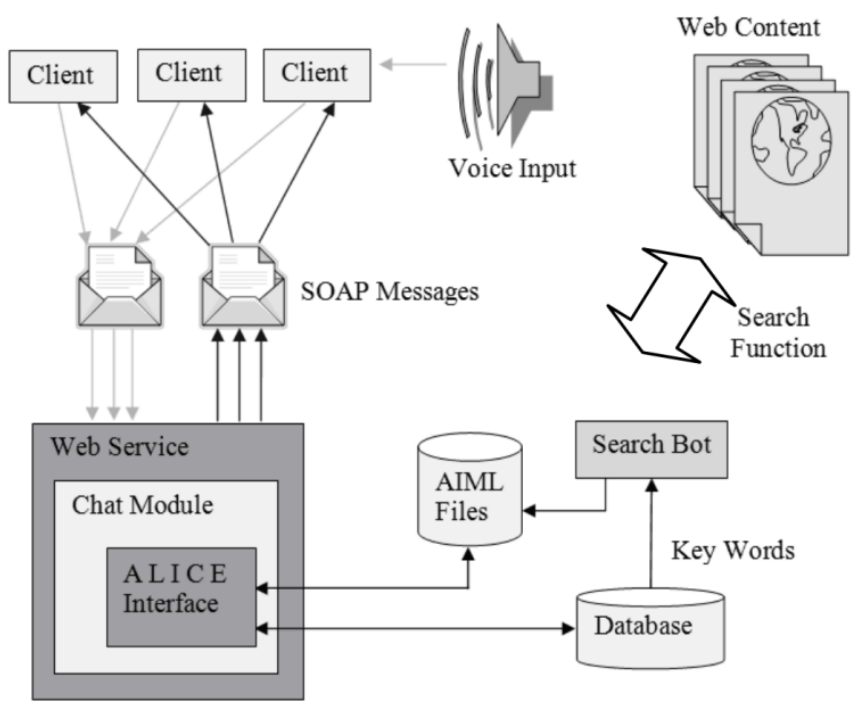

Fig. 1 - System architecture

All queries sent through the Web Service are processed by a program that generates responses (based on the Artificial Linguistic Internet Computing Entity (ALICE) system), which utilizes the data repository to the fullest extent possible. Content retrieval module updates the data repository automatically by using an Artificial Intelligence Markup Language (AIML) to increase the level of intelligence autonomously (based on the repository data). Since this approach entails the function of a third party administrator to find out on a regular basis whether the self-training function is of the highest 
quality. As and when needed, the content is reprocessed and incremental updates are supplied as and when they are required for future queries.

\section{SYSTEM IMPLEMENTATION}

JAVA was used in the development of the demonstrator for this study, and HTML was used to embed the applet. The JAVA component is hidden from the end-user to give the illusion of simplicity shown in Fig. 2. Apache web server hosts the website with the embedded applet. Open-source database management software, MySQL, is used to manage the database and website.

For processing voice inputs, the applet requires several libraries. Libraries signed by the developers were kept on the website hosting the applets, like the applets. Loading sequences are used to streamline the launch of libraries before the applet launches within a browser.

By pre-loading libraries, the operations are streamlined. The user is asked to accept a signature, upon which the applet is in a position to securely communicate with the web service. A digital signature can be created for applets and libraries using NetBeans, which is an open-source development environment. Applets can communicate with web services from sources other than their source web servers. User interactions activate the chat client, which is interrupt-driven. This is shown in Fig. 3. The control unit decides which function to execute next and which component to launch.

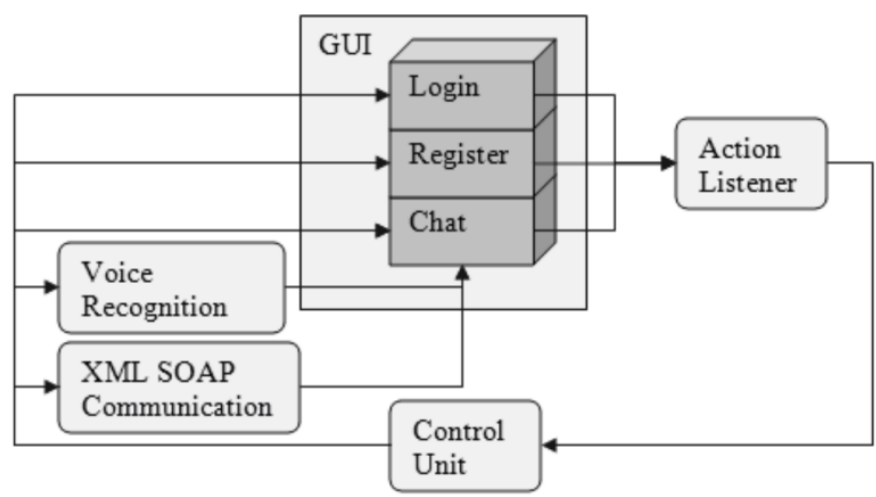

Fig. 2 - Applet management process
In the graphical user interface (GUI), the button listeners (login, register, and chat) are connected to the action listener. An incident triggers one of the SOAP communication modules, the voice recognition module, or the interface. User input is captured via the GUI for input and output in these modules.

The voice recognition process is carried out using an open-source library called Sphinx 4 . There are two types of speech recognition; speakers and speech styles. The speech styles of single and independent speakers include those that focus on isolated words, words connected by connections, and words which continue to exist throughout the speech process.

In order to recognize isolated words, breaks must be made between every word. It also requires considerably shorter breaks when word recognition is continuous. Last but not least, continuous speech recognition is a style in which a speaker speaks continuously without stopping or braking between words. This code has difficulty deciphering words that start with similar vowels. There are several components of the speech system that are important. The configuration file is one of those components. In the figure below, you can see that. As we have mentioned earlier, a configuration file is utilized as the starting point for writing the recogniser and (or) decoder from an application programming interface (API), which includes using the dictionary. In the API, there is also an acoustic model that contains grammar files that help generate the message.

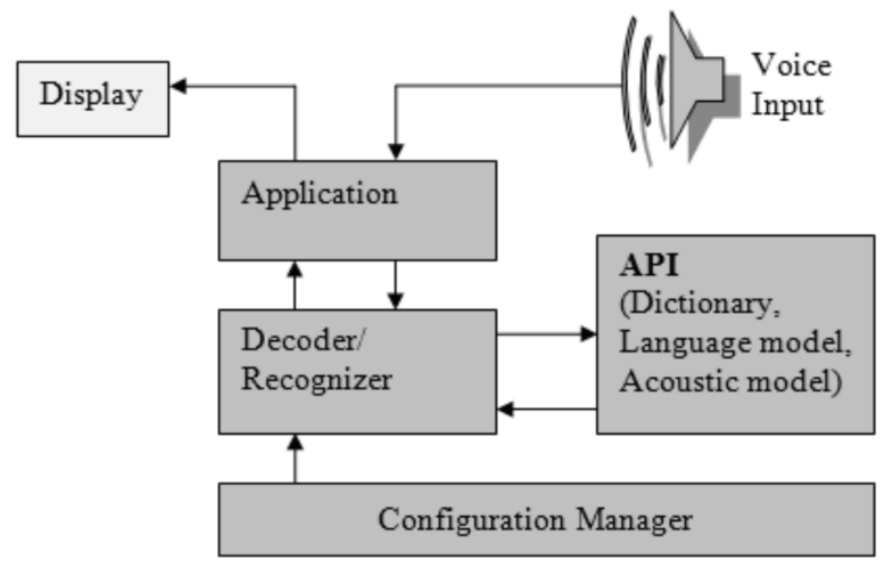

Fig. 3 - Speech recognition framework 
The speech framework consists of three main components: the front-end app, the decoder, and the language model. Voice input is acquired and attributed by the front-end. With the use of a dictionary and construction of words found in a lookup table (LUT), the language and acoustic model translates from a standard language (which is input to the system). Using the attributes and LUTs calculated from the input voice, the result set is produced by a search manager that is situated within the decoder after the voice has been decoded. Activating the configuration manager will load all components into XML, which can be accessed from the front end.

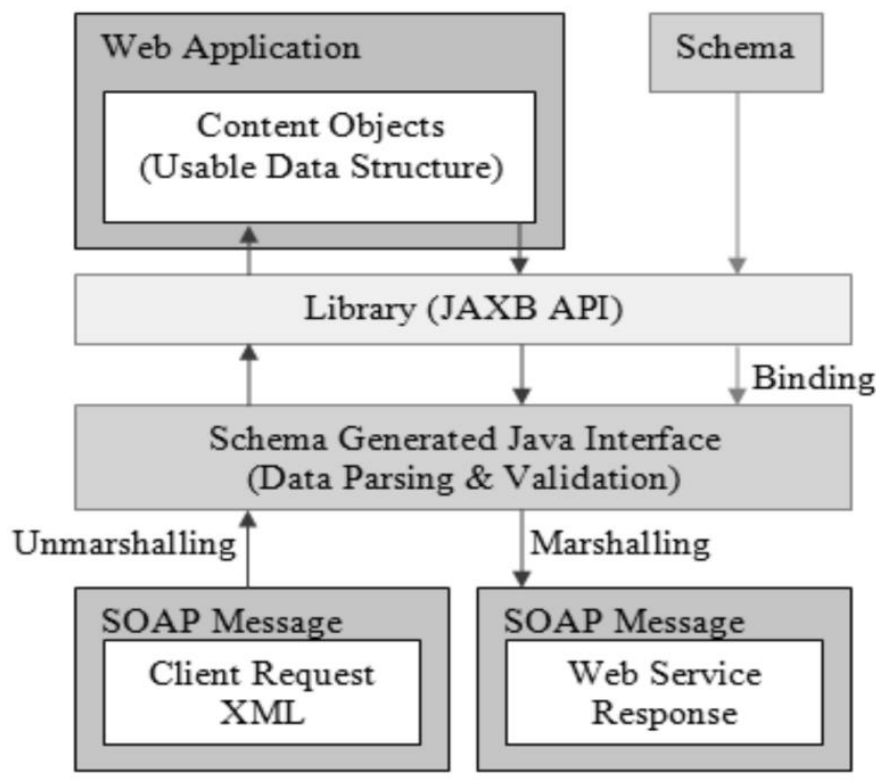

Fig. 4 - Distributed system framework

There is a truncated API (Pocketsphinx), focusing on the mobile platform, which is available for speechenabled applications. This paper explores a more toned-down API, Spinx 4, focusing on the embedded platform. A text-based and XML-formatted SOAP protocol is used in communication with the web service. An XML interface was created using a Javabased architecture for XML binding (JAXB). Other developers may also be able to develop XML schemabased APIs through a website. As shown in Fig. 6, you can see how the interface is generated.

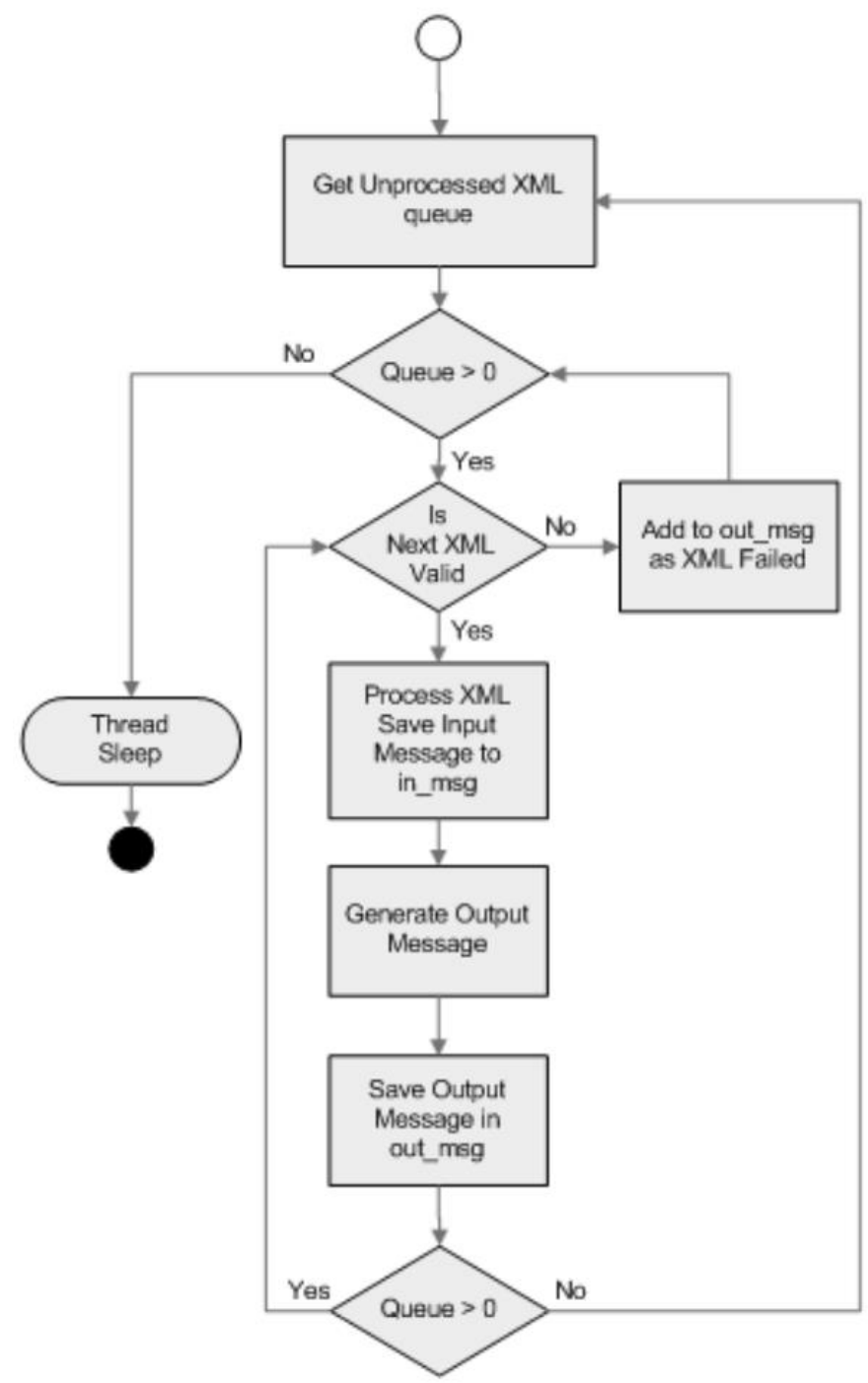

Fig. 5 - Object binding process

If an XML message is invalid, the interface generates a fault response. Once the error location has been determined by the bound object, it is encapsulated in $\mathrm{XML}$ and sent using SOAP. If the format of the message changes or the needs change with respect to the format of the message, then the interface can be regenerated.

It is possible to maintain a large number of users with the use of synchronized threads. HTTP (Hypertext Transfer Protocol) sockets are the most basic means of communication between clients or servers when requesting content. A client establishes a connection through a socket connection (socket connection), and the socket creates a replacement thread-duo (incoming, outgoing and processes) to deal with the 
client's messages. Once the socket is pulled, all messages are processed through the interface object.

Using the push-pop algorithm, all messages are queued by the thread pair. Fig. 7 shows how messages are pulled from the queue for processing in a controlled manner. As a result, processing times are optimized.

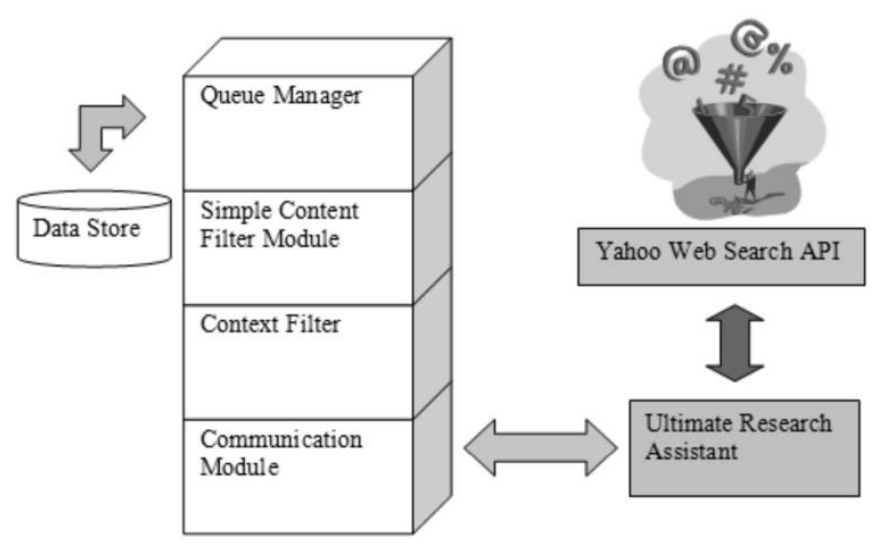

Fig. 6 - Processing thread

A training module was developed to aid in the training process by processing content obtained from the internet regarding statements or questions that the system cannot understand. For such a search to be conducted as well as provide concise content, a complex algorithm must be developed. An expert system, "Ultimate Research Assistant", was used to produce a detailed report on such a press release. In Fig. 8, we can see how the process works.

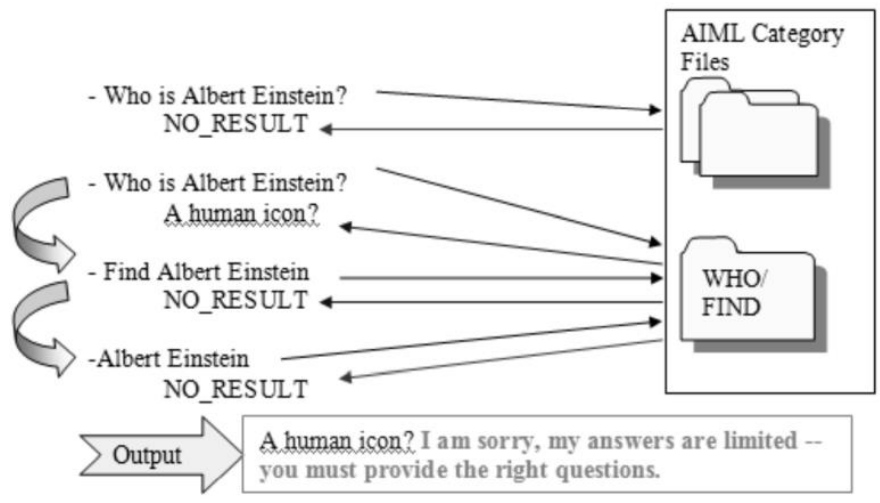

Fig. 7 - Content request utilizing the final word Research Assistant

It is essential to refine the query sent to the expert system so that it will provide the most relevant response. AIML-assisted categories are used for self- processing during query refinement. As an example, the query "Who is Albert Einstein?" may not yield a result (Fig. 9), indicating that the system uses the workflow described above and that the query is repeated iteratively in the secondary file(s). "A human icon" may be the result of this query, and the subsequent processing produces a response that includes the output needed from the secondary file along with a standard response ("I am sorry, please provide the right questions").

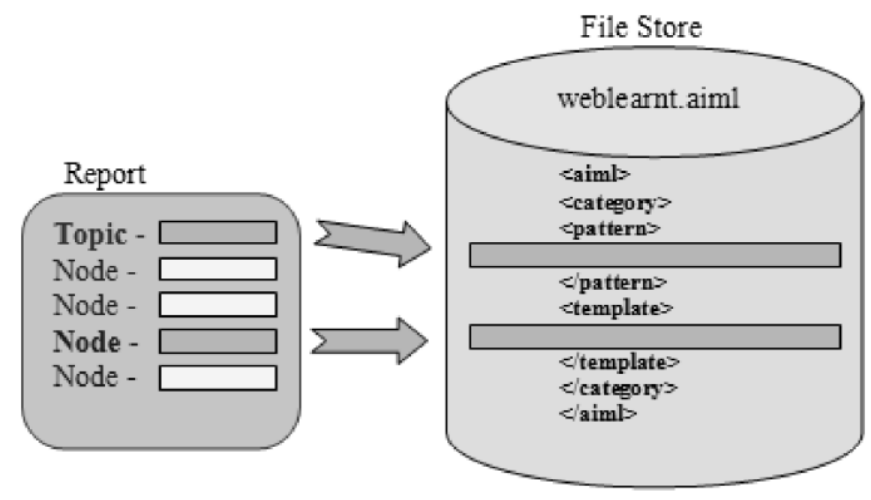

Fig. 8 - ALICE-based processing of statement/question using AIML-assisted categories As a result, "Albert Einstein" is understood in this instance, but also becomes the refined query. Once the refined query has been submitted, it is added to the queue for consideration by the expert system. The AIML files are further filled up as the queues are processed and reports are generated. This process is shown in Fig. 10. 


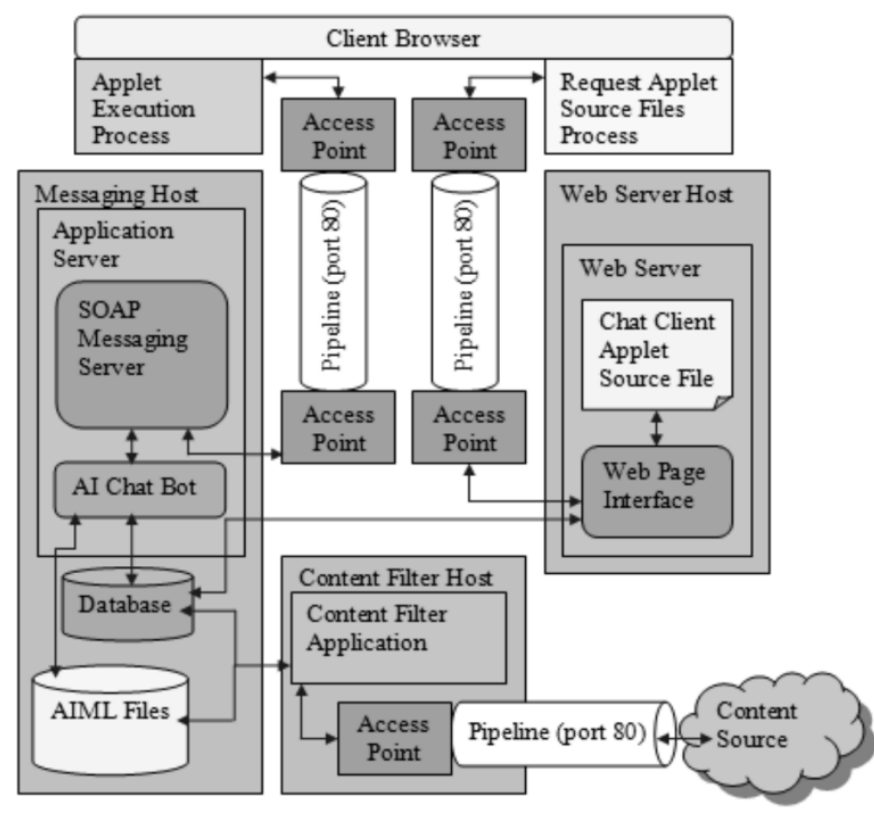

Fig. 9 - The process of populating AIML files

The process in Fig. 9 is autonomous and deserves minimal moderation to control AIML file expansion. As a result of this process, the intelligence of the system improves.

\section{RESULTS}

By combining voice input and output, a client can run on a variety of platforms and experience a simplified experience. Due to the internet-based nature of clients, thin-client systems or even mobile devices are next in line. Thick client systems are embedded computers that have limited processing capabilities and are controlled by a central server. You can use a minicomputer on your refrigerator or a GPS unit on your smartphone. The purpose of this work was to implement a virtual online friend that would be intelligent, as well as have spoken capabilities, but these underlying technologies can also be applied to other applications, particularly when needs are faced related to input control access or resource limitations.

The participants of the system were able to achieve an environment where resources were managed and stability could be achieved amongst modules. A schematic representation of this is shown in Fig. 10. Additionally, the chatbot will also host the website, conduct responses through the ALICE bot engine, and generate content via an expert system, the chatbot will gain autonomous intelligence by obtaining the necessary information from the site. Through the use of the distributed framework, the system can handle an increased number of users and increase its throughput. This paper's technology demonstrator may be limited in age by the lifetime of the expert system.

\section{CONCLUSION}

By creating a distributed system that is transparent and high performing, the overall system can be made transparent, through its modular design. Performance is determined by the total processing power available in the systems. Having all the modules running on different systems has reduced the possibility of overload, which is further reduced through the use of a chat client that communicates with a telephony server for voice processing.

Intelligent improvements are made possible using a comprehensive expert system (Ultimate Research Assistant). To increase the intelligence of ALICE-bots, conventional implementations included an administrator updating the AIML files manually. The information the expert system returns to us is already selected for relevance, so the information is only processed minimally. It is possible that our assessment of the situation is subjective since the intelligence was delegated to a third party. If this third party system were to be decommissioned, this could put us at risk as well. As a result of the implementation of threads, it is possible to process incoming and outgoing messages multiple times without having to wait for one message to be processed or for a server to become unavailable due to heavy usage or congestion. Whenever a server connection was made, a pair of new threads were created independent of other threads related to other users. A framework such as this can be used for more than just chat applications. The ability to build information systems on top of the 
existing framework is the most promising potential owing to its distributed nature. There are options for introducing additional components at the AIML level, as well as AIML file functionality, depending on the integration requirements.

\section{VII.REFERENCES}

[1]. Xuewen $\mathrm{Mu}$, Xiaoping Shen and John Kirby 2017 Support Vector Machine Classifier Based on Approximate Entropy Metric for Chatbot Text-based Communication International Journal of Artificial Intelligence ISSN: 09740635 vol 15 (2) pp 1-16

[2]. Rashmi Dharwadkar and Neeta A Deshpande 2018 A Medical Chatbots. International Journal of Computer Trends and Technology vol 60 (1) pp 41-45.

[3]. Divya S, Indumathi V, Ishwarya S, Priyasankari M and Kalpana Devi S 2018 A Self-Diagnosis Medical Chatbot Using Artificial Intelligence Journal of Web Development and Web Designing vol 3 (1) pp 1-7.

[4]. Imran Ahmed and Shikha Singh 2015 AIML Based Voice Enabled Artificial Intelligent Chatterbot International Journal of u-and eService, Science and Technology vol 8 (2) pp375-384.

[5]. Pryss R et al. 2019 Using Chatbots to Support Medical and Psychological Treatment Procedures: Challenges, Opportunities, Technologies, Reference Architecture. Baumeister H, Montag C (eds) Digital Phenotyping and Mobile Sensing Studies in Neuroscience, Psychology and Behavioral Economics Springer, Cham, ISBN 978-3-03031619-8 pp 249-260.

[6]. Divya Madhu, Neeraj Jain C J, Elmy Sebastain, Shinoy Shaji and Anandhu Ajayakumar 2017 A Novel Approach for Medical Assistance Using Trained Chatbot International Conference on
Inventive Communication and Computational Technologies (ICICCT) pp 243-246.

[7]. Amiya Kumar Tripathy, Rebeck Carvalho, Keshav Pawaskar and Suraj Yadav 2015 Mobile based healthcare management using artificial intelligence International Conference on Technologies for Sustainable Development (ICTSD) pp 4-6.

[8]. Sagar Gupta, Jenila Livingston L M and Agnel Livingston L G X 2019 Prediction of Top Tourist Attraction Spots using Learning Algorithms International Journal of Recent Technology and Engineering ISSN: 2277-3878 vol 8 (3) pp 10631067.

[9]. Lucas et al. 2020 A Machine Learning Framework to Predict Nutrient Content in Valencia Orange Leaf Hyperspectral Measurements Remote Sensing MDPI vol 12 (6) 906 doi:10.3390/rs12060906 pp 1-21.

[10]. Augello A. Saccone G. Gaglio S. Pilato G., Humorist Bot: Bringing Computational Humour in a Chat-Bot System. Proceedings of the International Conference on "Complex, Intelligent and Software Intensive Systems (CISIS)", 4-7 March 2008, Barcelona, Spain, pp.703-708.

[11]. Gambino O. Augello A. Caronia A. Pilato G. Pirrone R. Gaglio S., Virtual conversation with a real talking head. Proceedings of the Conference on "Human System Interactions", 25-27 May 2008, Kraow, Poland, pp. 263-268.

[12]. Vojtko J. Kacur J. Rozinaj G., The training of Slovak speech recognition system based on Sphinx 4 for GSM networks. Proceedings of International Symposium "EL, MAR (Electronics in Marine) focused on Mobile Multimedia", 12-14 Sept. 2007, Zadar, Croatia, pp. 147-150.

[13]. Carnegie Mellon University, Sun Microsystems, Mitsubishi Electric Research Laboratories, Sphinx-4 - A speech recognizer written entirely in the JAVA programming language, 2004. 
[14]. Carnegie Mellon University (CMU). Speech at CMU. http://www.speech.cs.cmu.edu

[15]. Rural India's access to healthcare patchy: Studyhttps://economictimes.indiatimes.com/ne ws/economy/indicators/rural-indias-access-tohealthcarepatchystudy/articleshow/21227645.cms, 2013

[16]. Raij, A.B., Johnsen, K., Dickerson, R.F., Lok, B.C., Cohen, M.S., Duerson, M., Pauly, R.R., Stevens, A.O., Wagner, P. and Lind, D.S., 2007. Comparing interpersonal interactions with a virtual human to those with a real human. IEEE transactions on visualization and computer graphics, 13(3), pp.443-457.

[17]. Fadhil, A., 2018."Beyond patient monitoring: Conversational agents role in telemedicine \& healthcare support for home-living elderly individuals". arXiv preprint arXiv:1803.06000.

[18]. Amato, F., Marrone, S., Moscato, V., Piantadosi, G., Picariello, A. and Sansone, C., 2017. Chatbots Meet eHealth: Automatizing Healthcare. In WAIAH@ AI* IA (pp. 40-49).

[19]. Comendador, BenildaEleonor V., et al. "Pharmabot: a pediatric generic medicine consultant chatbot." Journal of Atomation and Control Engineering Vol 3.2 (2015).

[20]. https://dialogflow.com/

[21]. https://firebase.google.com/docs/functions

[22]. Jacob, I. Jeena. "Performance Evaluation of Caps-Net Based Multitask Learning Architecture for Text Classification." Journal of Artificial Intelligence 2, no. 01 (2020): 1-10.

[23]. Herring, S.C.: Slouching Toward the Ordinary: Current Trends in Computer Mediated Communication. New Media \& Society 6, 26-36 (2004). [25] Smith, A., Page, D.: U.S. Smartphone Use in 2015. PewResearchCenter (2015).

[24]. Silva, B.M.C., Rodrigues, J.J.P.C., La Torre Diez, I.d., et al.: Mobile-health: A review of current state in 2015. Journal of biomedical informatics 56, 265-272 (2015). 